\title{
A CLASS OF NONSOFIC MULTIDIMENSIONAL SHIFT SPACES
}

\author{
RONNIE PAVLOV \\ (Communicated by Bryna Kra)
}

\begin{abstract}
In one dimension, sofic shifts are fairly well understood and are special examples of shift spaces which must satisfy very restrictive properties. However, in multiple dimensions there are very few known conditions which guarantee nonsoficity of a shift space. In this paper, we show that for any $\mathbb{Z}^{d}$ sofic shift $X$ which satisfies a uniform mixing condition called block gluing in all directions $\overrightarrow{e_{2}}, \ldots, \overrightarrow{e_{d}}$, the set of legal rows of $X$ in the $\overrightarrow{e_{1}}$-direction has a synchronizing word. This allows us to define a (new) large class of nonsofic $\mathbb{Z}^{d}$ shift spaces.
\end{abstract}

\section{INTRODUCTION}

The field of topological dynamics is concerned with the study of topological dynamical systems; a topological dynamical system is comprised of a compact space $X$ and a group action $\left(T_{g}\right)_{g \in G}$ of self-homeomorphisms of $X$. (In this paper, $G$ will always be $\mathbb{Z}^{d}$ for some $d$.)

Symbolic dynamics is devoted to the study of a specific type of topological dynamical system called a shift space, defined by a finite set $A$ (called an alphabet) and a (possibly infinite) set $\mathcal{F}$ of functions from finite subsets of $\mathbb{Z}^{d}$ to $A$, called configurations. The $\mathbb{Z}^{d}$ shift space $X_{\mathcal{F}}$ induced by $\mathcal{F}$ is then the set of all $x \in A^{\mathbb{Z}^{d}}$ which contain no translate of any of the configurations from $\mathcal{F}$. We always think of $A^{\mathbb{Z}^{d}}$ as being endowed with the (discrete) product topology, and any $\mathbb{Z}^{d}$ shift space as a topological space with the induced topology. Any shift space is a topological dynamical system when acted on by the $\mathbb{Z}^{d}$ shift action of translations by vectors in $\mathbb{Z}^{d}$.

A specific type of well-studied shift space occurs when $\mathcal{F}$ is finite. In this case, we call $X_{\mathcal{F}}$ a $\mathbb{Z}^{d}$ shift of finite type, or SFT. A slightly more general class of shift spaces are the $\mathbb{Z}^{d}$ sofic shifts, which are the images of SFTs under shift-commuting continuous maps.

In one dimension, SFTs and sofic shifts are fairly well behaved and satisfy many restrictive properties. For example, under the fairly mild assumption of topological mixing, any one-dimensional SFT or sofic shift has dense periodic points, topological entropy equal to the log of an algebraic number, and a unique measure of maximal entropy. (We postpone the formal definition of topological entropy until Section 2, for now, we only note that it is an isomorphism invariant assigned to any shift space $X$ which in some sense measures the "information" contained in $X$. We will not define the notions of topological mixing or measure of maximal entropy in this

Received by the editors March 25, 2011 and, in revised form, August 2, 2011.

2010 Mathematics Subject Classification. Primary 37B50; Secondary 37B10, 37A15.

Key words and phrases. $\mathbb{Z}^{d}$, shift of finite type, sofic, multidimensional. 
paper; see [13] for more information.) There also exist algorithmically checkable necessary and sufficient conditions for being a $\mathbb{Z}$ SFT or sofic shift.

In $\mathbb{Z}^{d}$, the situation is much different; sofic shifts and SFTs seem to be much more difficult objects to study. For instance, the question of whether a $\mathbb{Z}^{d}$ SFT is even nonempty is algorithmically undecidable given the forbidden configurations (2], 14 ). It is quite easy, however, to give examples of $\mathbb{Z}^{d}$ shift spaces which are not SFTs. For example, consider the $\mathbb{Z}^{d}$ shift space $X$ on the alphabet $\{0,1\}$ consisting of all $\omega \in\{0,1\}^{\mathbb{Z}^{d}}$ which have either zero or one occurrence of the symbol 1 . Then $X$ is not an SFT: suppose for a contradiction that it is an SFT with $\mathcal{F}$ contained in $\{0,1\}^{\{1, \ldots, n\}^{d}}$ for some $n$. Then consider any $\omega \in\{0,1\}^{\mathbb{Z}^{d}}$ which contains two 1 symbols, separated by a distance of more than $n$. Clearly $\omega$ does not contain any configuration from $\mathcal{F}$, but by definition $\omega \notin X$. This contradicts the assumption that $X=X_{\mathcal{F}}$.

The situation with sofic shifts is different; it seems difficult to prove that a $\mathbb{Z}^{d}$ shift is nonsofic, since a $\mathbb{Z}^{d}$ sofic shift might be induced by a factor map from an SFT with a much larger alphabet which loses a great deal of information.

There are (at least) two sufficient conditions in the literature for nonsoficity. Firstly, it was shown in [7] that any $\mathbb{Z}^{d}$ sofic shift $S$ must satisfy a condition called effectiveness, which means that there exists a Turing machine $T$ which, on input $n$, outputs a configuration $u^{(n)}$, such that the collection $\left\{u^{(n)}\right\}$ is a set of forbidden configurations inducing the shift space $S$, e.g. $S=\left\{x \in A^{\mathbb{Z}^{d}}\right.$ : $x$ does not contain any $\left.u^{(n)}\right\}$. Therefore, any noneffective shift space must be nonsofic. However, this condition is not so helpful for giving explicit examples of nonsofic shift spaces; most shift spaces in the literature have algorithmic descriptions, meaning that they are effective.

Secondly, it was shown in 4 that any $\mathbb{Z}^{d}$ sofic shift $S$ with positive topological entropy $h(S)$ contains proper subsystems of topological entropy arbitrarily close to $h(S)$. As an application, we note that if a $\mathbb{Z}^{d}$ shift space $X$ has positive topological entropy and is minimal, meaning that it contains no proper nonempty subshift, then this result implies that $X$ is nonsofic. (See [8] for a method of constructing such shift spaces.)

Our main result is a new sufficient condition for nonsoficity of a $\mathbb{Z}^{d}$ shift space $X$.

Theorem 1.1. If $Z$ is a $\mathbb{Z}^{d}$ shift space on the alphabet $A$ which is block gluing in the directions $\overrightarrow{e_{2}}, \ldots, \overrightarrow{e_{d}}$, and if the $\mathbb{Z}$ shift space $\left\{\left.z\right|_{\mathbb{Z} \times \vec{v}}: z \in Z, \vec{v} \in \mathbb{Z}^{d-1}\right\}$ consisting of all rows of points of $Z$ in the $\overrightarrow{e_{1}}$-direction does not have a synchronizing word, then $Z$ is not sofic.

(Here and in the rest of the paper, $\left\{\vec{e}_{i}\right\}$ is the standard collection of basis vectors in $\mathbb{Z}^{d}$.) We postpone rigorous definitions until the next section, but will briefly address the two hypotheses of the theorem.

Block gluing, informally speaking, is a condition that allows very large rectangular configurations which appear in some points of $X$ (possibly different ones) to coexist in a single point of $X$ as long as they are separated by a certain distance, which does not depend on the size of the configurations chosen.

A synchronizing word for a $\mathbb{Z}$ shift space $X$ is essentially a word $w$ for which the possible words which can appear to the left and right of $w$ in a point of $X$ are independent. More rigorously, $w$ is synchronizing if for any words $u$ and $v$ for which 
the concatenations $u w$ and $w v$ appear in some points of $X$, the concatenation $u w v$ appears in some point of $X$. It is well known that $\mathbb{Z}$ sofic shifts and SFTs have synchronizing words (in fact, for any $\mathbb{Z} \mathrm{SFT}$, all sufficiently long words are synchronizing!), and so our assumption that the set of rows of $X$ has no synchronizing words is simply a condition guaranteeing that the set of rows of $X$ is far from being sofic.

Our result gives at least one way to generate a large class of new nonsofic $\mathbb{Z}^{d}$ shift spaces. First, choose any $\mathbb{Z}$ shift space $Y$ which is effective and has no synchronizing word, for example a Sturmian shift space with computable rotation number. (See [6], Chapter 6, for an introduction to Sturmian shift spaces.) Then, define $X$ to be the independent product of $Y$ along the directions $\overrightarrow{e_{2}}, \ldots, \overrightarrow{e_{d}}$, i.e. $X:=\{x \in$ $\left.A^{\mathbb{Z}^{d}}: \forall \vec{v} \in \mathbb{Z}^{d-1}, \ldots x(-1, \vec{v}) x(0, \vec{v}) x(1, \vec{v}) \ldots \in Y\right\}$. Then $X$ is block gluing in those directions and so nonsofic by our main result, but we claim that it does not satisfy either of the aforementioned existing conditions for nonsoficity.

Firstly, the fact that $Y$ is effective implies that $X$ is effective. Secondly, $X$ contains subsystems with topological entropy arbitrarily close to $h(X)$ : for any $k$, define $X_{k}$ to be the subsystem of $X$ consisting of points in $X$ for which there exists $i \in[0, k)$ such that $\left.x\right|_{\mathbb{Z}^{d-1} \times\{j\}}=\left.x\right|_{\mathbb{Z}^{d-1} \times\{i\}}$ for every $j \equiv i(\bmod k)$. In other words, for each point in $X_{k}$, every $k$ th $(d-1)$-dimensional hyperplane in the $\overrightarrow{e_{d}}$-direction is the same. It is then easily checked that $h\left(X_{k}\right)=\frac{k-1}{k} h(X)$.

Theorem 1.1 is also related to results about subdynamics of $\mathbb{Z}^{d}$ sofic shifts, such as the following result, paraphrased slightly from comments following Theorem 1.4 from [7].

Theorem $1.2\left([7)\right.$. For any effective $\mathbb{Z}$ shift space $X$, there exists a $\mathbb{Z}^{3}$ sofic shift $\widetilde{X}$ such that $X$ is the set of rows of $\widetilde{X}$ in the $\overrightarrow{e_{1}}$-direction. The effective $\mathbb{Z}$ shift spaces are the only ones which can arise in this way.

Two recent preprints $([1],[5])$ extend this result to $\mathbb{Z}^{2}$. These results all imply that an incredibly diverse class of $\mathbb{Z}$ shift spaces can be realized as the rows of a $\mathbb{Z}^{d}$ sofic shift. However, their examples are very deterministic (in fact, constant!) along the other cardinal directions. Theorem 1.1 can then be thought of as a weak counterpoint: if one wishes to realize sufficiently esoteric shift spaces in the $\overrightarrow{e_{1}}$ direction (such as a shift space without a synchronizing word), then at least some determinism is necessary along another direction.

\section{DEFINITIONS AND PRELIMINARIES}

Definition 2.1. A configuration in $\mathbb{Z}^{d}$ over a finite alphabet $A$ is a function $w$ from a finite set $S \subset \mathbb{Z}^{d}$, called the shape of $w$, to $A$. A word is a configuration in $\mathbb{Z}$ whose shape is $[m, n]$ for some $m \leq n \in \mathbb{Z}$. (Here and elsewhere, for any $a \leq b \in \mathbb{Z}$, $[a, b]$ refers to the interval of integers $\{a, a+1, \ldots, b\}$.)

For any $d$ and alphabet $A$, we denote by $A^{*}$ the set of configurations in $\mathbb{Z}^{d}$ over $A$.

Definition 2.2. A point $x \in A^{\mathbb{Z}^{d}}$ contains a configuration $w \in A^{S}$ if there exists $\vec{v} \in \mathbb{Z}^{d}$ such that $x(\vec{u}+\vec{v})=w(\vec{u})$ for all $\vec{u} \in S$.

Definition 2.3. For any $d$ and set $\mathcal{F} \subseteq A^{*}$, we say that $\mathcal{F}$ induces a $\mathbb{Z}^{d}$ shift space $X_{\mathcal{F}}:=\left\{x \in A^{\mathbb{Z}^{d}}:\right.$ no configuration in $\mathcal{F}$ appears in $\left.x\right\}$. 
We repeat that shift spaces are compact topological spaces when endowed with the induced product topology from $A^{\mathbb{Z}^{d}}$. They are also topological dynamical systems under the $\mathbb{Z}^{d}$ shift action $\sigma_{\vec{v}}$ by homeomorphisms, defined by $\left(\sigma_{\vec{v}}(x)\right)(\vec{u})=$ $x(\vec{u}+\vec{v})$ for any $x \in A^{\mathbb{Z}^{d}}$.

Definition 2.4. For any $d$, a $\mathbb{Z}^{d}$ shift of finite type or $S F T$ is a shift space $X_{\mathcal{F}}$ induced by a finite set $\mathcal{F}$ of forbidden configurations. If all configurations in $\mathcal{F}$ are adjacent pairs of letters, then $X_{\mathcal{F}}$ is called a $\mathbb{Z}^{d}$ nearest neighbor SFT.

Definition 2.5. A $\mathbb{Z}^{d}$ factor map is a continuous map $\phi$ from a $\mathbb{Z}^{d}$ shift space $X$ to a $\mathbb{Z}^{d}$ shift space $Y$ which is shift-commuting; i.e. $\sigma_{\vec{v}}(\phi(x))=\phi\left(\sigma_{\vec{v}}(x)\right)$ for any $x \in X$ and $\vec{v} \in \mathbb{Z}^{d}$.

Definition 2.6. A $\mathbb{Z}^{d}$ sofic shift is the image of a $\mathbb{Z}^{d}$ SFT under a $\mathbb{Z}^{d}$ factor map.

In fact, given a $\mathbb{Z}^{d}$ sofic shift $S$, it is well known that there exists a $\mathbb{Z}^{d}$ nearest neighbor SFT $X$ and a factor map $\phi$ such that $S=\phi(X)$, and $\phi$ is a letter-to-letter map; i.e. for any $x \in X$ and $\vec{v} \in \mathbb{Z}^{d},(\phi(x))(\vec{v})$ depends only on $x(\vec{v})$.

Definition 2.7. For any $\mathbb{Z}^{d}$ shift space $X$ on the alphabet $A$, the language of $X$ is $L(X):=\left\{w \in A^{*}: \exists x \in X\right.$ containing $\left.w\right\}$. For any finite $S \subset \mathbb{Z}^{d}$, we make the notation $L_{S}(X)=L(X) \cap A^{S}$.

Definition 2.8. The topological entropy of a $\mathbb{Z}^{d}$ shift space $X$ is

$$
h(X):=\lim _{n \rightarrow \infty} \frac{1}{n^{d}} \log \left|L_{[1, n]^{d}}(X)\right| .
$$

For the next five definitions, for words $u, v$, we use $u v$ to denote the concatenation of $u$ and $v$.

Definition 2.9. For any $\mathbb{Z}$ shift space $X$ and any word $w \in L(X)$, the follower set of $w$ is $F(w):=\{v \in L(X): w v \in L(X)\}$.

Definition 2.10. For any $\mathbb{Z}$ shift space $X$ and any word $w \in L(X)$, the predecessor set of $w$ is $P(w):=\{v \in L(X): v w \in L(X)\}$.

Definition 2.11. For any $\mathbb{Z}$ shift space $X$, a word $w \in L(X)$ is synchronizing if for all $u, v \in L(X)$ for which $u w$ and $w v$ are in $L(X)$, it is also the case that $u w v \in L(X)$.

It follows immediately from the definition that $w$ is synchronizing if and only if $P(w)=P(w v)$ for all $v$ for which $w v \in L(X)$.

Definition 2.12. For any finite alphabet $A$, a set $S \subseteq A^{*}$ is left-factorial if for any $v, w \in A^{*}$, if $v w \in S$, then $v \in S$.

Definition 2.13. For any finite alphabet $A$, a set $S \subseteq A^{*}$ is left-extendable if for any $w \in S$, there exists $v \in A^{*}$ so that $v w \in S$.

It is easily checked that for any $\mathbb{Z}$ shift space $X, L(X)$ is left-factorial and leftextendable.

Definition 2.14. A $\mathbb{Z}^{d}$ shift space $X$ is block gluing in direction $\overrightarrow{e_{i}}$ (with filling length $L$ ) if for any rectangular prisms $R, R^{\prime}$ with $\min _{\vec{r} \in R, \vec{r}^{\prime} \in R^{\prime}}\left|r_{i}-r_{i}^{\prime}\right| \geq L$, and for any configurations $u \in L_{R}(X)$ and $u^{\prime} \in L_{R^{\prime}}(X)$, there exists $x \in X$ with $\left.x\right|_{R}=u$ and $\left.x\right|_{R^{\prime}}=u^{\prime}$. 
In [3] and [12, a shift space is said to be block gluing if it is block gluing in every cardinal direction $\overrightarrow{e_{1}}, \ldots, \overrightarrow{e_{d}}$.

Definition 2.15. For any $\mathbb{Z}^{d}$ shift space $X$ on the alphabet $A$, the $\mathbb{Z}$-projective subdynamics of $X$, denoted by $P_{\mathbb{Z}}(X)$, is the set of bi-infinite sequences in the $\overrightarrow{e_{1}}$-direction appearing in points of $X$.

Projective subdynamics were first defined in [10]. They were further studied in [12], where, among other things, the class of sofic shift spaces realizable as $P_{\mathbb{Z}}(X)$ for a $\mathbb{Z}^{2}$ SFT $X$ was determined.

Definition 2.16. $\vec{u}, \vec{v} \in \mathbb{Z}^{d}$ are adjacent if $\sum_{i=1}^{d}\left|u_{i}-v_{i}\right|=1$.

Definition 2.17. For any finite set $S \subset \mathbb{Z}^{d}$, the boundary of $S$, written $\partial S$, is the set of $\vec{u} \in S$ adjacent to some $\vec{v} \in \mathbb{Z}^{d} \backslash S$.

Note that our definition of $\partial S$ is an "inner" boundary, meaning that $\partial S \subseteq S$. Many authors use $\partial S$ to refer to the set of sites outside $S$ but adjacent to a site within $S$, which is $\partial\left(S^{c}\right)$ in our notation.

\section{Proof of Theorem 1.1}

We for now assume that $d=2$. Take any $\mathbb{Z}^{2}$ shift space $Z$ with alphabet $A$ as in the statement of Theorem 1.1, denote by $L$ an integer greater than the filling length of $Z$ in the $\overrightarrow{e_{2}}$-direction, and define $X=P_{\mathbb{Z}}(Z)$. By assumption, $X$ has no synchronizing word. Choose any $w^{(1)} \in L(X)$. Since $w^{(1)}$ is not synchronizing, there exists $w^{(2)} \in L(X)$ so that $w^{(1)} w^{(2)} \in L(X)$ and $P\left(w^{(1)} w^{(2)}\right) \subsetneq$ $P\left(w^{(1)}\right)$. Since $w^{(1)} w^{(2)}$ is not synchronizing, there exists $w^{(3)} \in L(X)$ so that $w^{(1)} w^{(2)} w^{(3)} \in L(X)$ and $P\left(w^{(1)} w^{(2)} w^{(3)}\right) \subsetneq P\left(w^{(1)} w^{(2)}\right)$. We can continue in this fashion to define words $w^{(1)}, w^{(2)}, \ldots$ such that $w^{(1)} \ldots w^{(n)} \in L(X)$ for all $n>0$, and $P\left(w^{(1)} \ldots w^{(n)} w^{(n+1)}\right) \subsetneq P\left(w^{(1)} \ldots w^{(n)}\right)$ for all $n>0$. For every $n$, choose $\bar{x}^{(n)} \in P\left(w^{(1)} \ldots w^{(n)}\right) \backslash P\left(w^{(1)} \ldots w^{(n)} w^{(n+1)}\right)$. Then for all $n>0$, $w^{(1)} \ldots w^{(n)} \in F\left(\bar{x}^{(n)}\right)$, but $w^{(1)} \ldots w^{(n)} w^{(n+1)} \notin F\left(\bar{x}^{(n)}\right)$.

For ease of notation, we define $y^{(i)}=w^{(1)} w^{(2)} \ldots w^{(i)}$ for any $i \in \mathbb{N}$. For any $m \in \mathbb{N}$, if $N_{m}=\max \left(\left\{\left|\bar{x}^{(i)}\right|\right\}_{i \in[1, m]}\right)$, then we can use left-extendability of $L(X)$ to extend each word $\left\{\bar{x}^{(i)}\right\}_{i \in[1, m]}$ on the left to words $\left\{x^{(m, i)}\right\}_{i \in[1, m]}$ of common length $N_{m}$ sharing the defining property of $\bar{x}^{(i)} ; y^{(i)} \in F\left(x^{(m, i)}\right)$, but $y^{(i+1)} \notin F\left(x^{(m, i)}\right)$. Since $L(X)$ is left-factorial and $y^{(i)}$ is a prefix of $y^{(i+1)}$ for every $i \in[1, m-1]$, for any $i, j \in[1, m], y^{(j)} \in F\left(x^{(m, i)}\right)$ iff $j \leq i$.

Suppose for a contradiction that $Z$ is sofic. Then there exists a $\mathbb{Z}^{2}$ nearest neighbor SFT $Y$ and letter-to-letter factor map $\phi$ so that $\phi(Y)=Z$. Define $k$ to be the size of the alphabet $B$ of $Y$, and as above define a set $\left\{x^{\left(k^{2 L}+1, i\right)}\right\}_{i \in\left[1, k^{2 L}+1\right]}$ of words of common length $N:=N_{k^{2 L}+1}$ and another set of words $\left\{y^{(i)}\right\}_{i \in\left[1, k^{2 L}+1\right]}$ such that for all $i, j \in\left[1, k^{2 L}+1\right], y^{(j)} \in F\left(x^{\left(k^{2 L}+1, i\right)}\right)$ iff $j \leq i$.

We need one new notation: for the remainder of the proof, for any $b \in \mathbb{N}$ and words $u^{(1)}, \ldots u^{(b)} \in A^{*}$ with lengths $a_{1}, \ldots, a_{b}$ respectively, we identify the $b$-tuple $\left(u^{(1)}, u^{(2)}, \ldots, u^{(b)}\right)$ with the configuration with shape $\bigcup_{i=1}^{b}\left(\left[1, a_{i}\right] \times\{L i\}\right)$ whose (Li)th row is $u^{(i)}$.

In this notation, for any $p \in \mathbb{N}$, define the collection $S_{p}=\left(\left\{x^{\left(k^{2 L}+1, i\right)}\right\}_{i \in\left[1, k^{2 L}+1\right]}\right)^{p}$ $=\left\{\left(x^{\left(k^{2 L}+1, i_{1}\right)}, \ldots, x^{\left(k^{2 L}+1, i_{p}\right)}\right): 1 \leq i_{1}, \ldots, i_{p} \leq k^{2 L}+1\right\}$ consisting of configurations made up of $p$ words from the set $\left\{x^{\left(k^{2 L}+1, i\right)}\right\}_{i \in\left[1, k^{2 L}+1\right]}$, separated by bands of 
$L-1$ rows. Since each of the words $x^{\left(k^{2 L}+1, i\right)}$ is in $L(X) \subset L(Z)$, by block gluing of $Z$ in the $\overrightarrow{e_{2}}$-direction, each configuration in $S_{p}$ is also in $L(Z)$.

Next, for any $w \in S_{p}$, define

$$
B(w)=\left\{\left.v\right|_{\partial([1, N] \times[1, L p])}: v \in L_{[1, N] \times[1, L p]}(Y),\left.\phi(v)\right|_{\bigcup_{i=1}^{p}([1, N] \times[L i])}=w\right\},
$$

the set of boundaries of extensions of $\phi$-preimages of $w$ to legal configurations (in $Y)$ on $[1, N] \times[1, L p]$.

For any $p \in \mathbb{N}$, define $D_{p}=L_{\partial([1, N] \times[1, L p])}(Y)$. Finally, for any $\delta \in D_{p}$, define the (possibly empty) set of configurations $U(\delta) \subseteq\left(\left\{y^{(i)}\right\}_{i \in\left[1, k^{2 L}+1\right]}\right)^{p}$ by $\left(y^{\left(i_{1}\right)}, \ldots, y^{\left(i_{p}\right)}\right) \in U(\delta)$ iff there exists $z \in Y$ so that $\left.z\right|_{\partial([1, N] \times[1, L p])}=\delta$ and, for each $1 \leq j \leq p, \phi\left(\left.z\right|_{\left[N+1, N+\left|y^{\left(i_{j}\right)}\right|\right] \times\{L j\}}\right)=y^{\left(i_{j}\right)} . U(\delta)$ is then the set of $p$-tuples of words from the set $\left\{y^{(i)}\right\}_{i \in\left[1, k^{2 L}+1\right]}$ which have $\phi$-preimages which, when separated by spaces of $L-1$ rows, can simultaneously "follow" $\delta$ on the right in some configuration in $L(Y)$. For example, if the configuration in Figure 1 is in $L(Y)$, and if each $\phi\left(u^{(j)}\right)$ is in the set $\left\{y^{(j)}\right\}_{j \in\left[1, k^{2 L}+1\right]}$, then $\left(\phi\left(u^{(1)}\right), \phi\left(u^{(2)}\right), \ldots, \phi\left(u^{(p)}\right)\right) \in U(\delta)$.

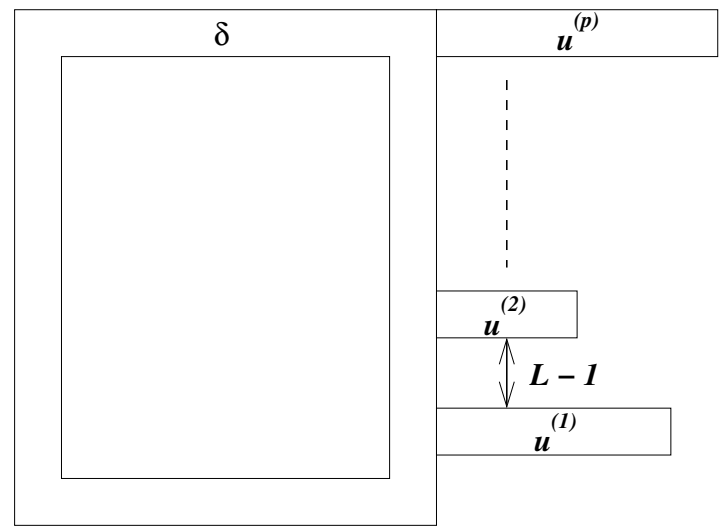

Figure 1. A configuration in $L(Y)$ demonstrating that $\left(\phi\left(u^{(1)}\right), \phi\left(u^{(2)}\right), \ldots, \phi\left(u^{(p)}\right)\right) \in U(\delta)$

Since $Y$ is a nearest neighbor SFT, for any rectangular configuration $v \in L_{R}(Y)$, the set of configurations on $\mathbb{Z}^{2} \backslash R$ which can be combined with $v$ to make a legal point of $Y$ depends only on the boundary of $v$. This means that for any $w \in S_{p}$, $\bigcup_{\delta \in B(w)} U(\delta)$ is just the set of all $p$-tuples of words from $\left\{y^{(i)}\right\}_{i \in\left[1, k^{2 L}+1\right]}$, separated vertically by bands of $L-1$ rows, which can "follow" $w$ on the right in some configuration in $L(Z)$.

If $w=\prod_{j=1}^{p} x^{\left(k^{2 L}+1, i_{j}\right)}$, then we claim that this set is $\prod_{j=1}^{p}\left(F\left(x^{\left(k^{2 L}+1, i_{j}\right)}\right) \cap\right.$ $\left.\left\{y^{(i)}\right\}_{i \in\left[1, k^{2 L}+1\right]}\right)$ : if $\left(y^{\left(k_{j}\right)}\right)_{j \in[1, p]}$ can "follow" $w$ on the right in some configuration in $L(Z)$, then clearly each $y^{\left(k_{j}\right)}$ is in $F\left(x^{\left(k^{2 L}+1, i_{j}\right)}\right)$. Also, for any configuration $\left(y^{\left(k_{j}\right)}\right)_{j \in[1, p]} \in \prod_{j=1}^{p} F\left(x^{\left(k^{2 L}+1, i_{j}\right)}\right)$, each word $x^{\left(k^{2 L}+1, i_{j}\right)} y^{\left(k_{j}\right)}$ is in $L(Z)$, and so the configuration $\left(x^{\left(k^{2 L}+1, i_{1}\right)} y^{\left(k_{1}\right)}, \ldots, x^{\left(k^{2 L}+1, i_{p}\right)} y^{\left(k_{p}\right)}\right)$ is also in $L(Z)$, by block gluing of $Z$ in the $\overrightarrow{e_{2}}$-direction. We simplify further by recalling that $F\left(x^{\left(k^{2 L}+1, i_{j}\right)}\right) \cap$ $\left\{y^{(i)}\right\}_{i \in\left[1, k^{2 L}+1\right]}=\left\{y^{(i)}\right\}_{i \in\left[1, i_{j}\right]}$. 
Lexicographically order the set of all $\left(i_{j}\right) \in\left[1, k^{2 L}+1\right]^{p}$, denoting the $r$ th such $p$-tuple by $\left(i_{j}^{(r)}\right)$. We have shown that for any $r \in\left[1,\left(k^{2 L}+1\right)^{p}\right]$, there exists $B_{r} \subseteq D_{p}\left(B_{r}=B\left(\prod_{j=1}^{p} x^{\left(k^{2 L}+1, i_{j}^{(r)}\right)}\right)\right)$ so that $\bigcup_{\delta \in B_{r}} U(\delta)=\prod_{j=1}^{p}\left\{y^{(i)}\right\}_{i \in\left[1, i_{j}^{(r)}\right.}$. Choose any $r>r^{\prime} \in\left[1,\left(k^{2 L}+1\right)^{p}\right]$.

Then by the definition of lexicographic order, for some $j^{\prime} \in[1, p], i_{j^{\prime}}^{(r)}>i_{j^{\prime}}^{\left(r^{\prime}\right)}$, and so the configuration $\left(y^{\left(i_{j}^{(r)}\right)}\right)_{j \in[1, p]}$ is in $\prod_{j=1}^{p}\left\{y^{(i)}\right\}_{i \in\left[1, i_{j}^{(r)}\right]} \backslash \prod_{j=1}^{p}\left\{y^{(i)}\right\}_{i \in\left[1, i_{j}^{\left(r^{\prime}\right)}\right]^{\prime}}$. Since $r^{\prime}$ was arbitrary between 1 and $r-1$, this implies that

$$
\prod_{j=1}^{p}\left\{y^{(i)}\right\}_{i \in\left[1, i_{j}^{(r)}\right]} \backslash \bigcup_{r^{\prime}=1}^{r-1} \prod_{j=1}^{p}\left\{y^{(i)}\right\}_{i \in\left[1, i_{j}^{\left(r^{\prime}\right)}\right]} \neq \varnothing
$$

which clearly implies that $B_{r} \backslash \bigcup_{r^{\prime}=1}^{r-1} B_{r^{\prime}} \neq \varnothing$ for all $r \in\left[2,\left(k^{2 L}+1\right)^{p}\right]$, and so $\left|\bigcup_{i=1}^{\left(k^{2 L}+1\right)^{p}} B_{i}\right| \geq\left(k^{2 L}+1\right)^{p}$. Since $\bigcup_{i=1}^{\left(k^{2 L}+1\right)^{p}} B_{i} \subset D_{p},\left|D_{p}\right| \geq\left(k^{2 L}+1\right)^{p}$. However, for any $p, D_{p}$ consists of configurations in $L_{\partial([1, N] \times[1, L p])}(Y)$, so $\left|D_{p}\right| \leq$ $k^{|\partial([1, N] \times[1, L p])|}<k^{2 L p+2 N}$. We then have a contradiction for large enough $p$, meaning that our original assumption was wrong and $Z$ is not sofic.

The proof for $d>2$ is similar, and we leave the details to the reader; the only change is that one must use the block gluing of $Z$ in the $\overrightarrow{e_{2}}, \ldots, \overrightarrow{e_{d}}$-directions to create $(d-1)$-dimensional grids of words from $\left\{y^{(i)}\right\}_{i \in\left[1, k^{2 L}+1\right]}$, separated by distances of $L-1$, rather than the one-dimensional "stacks" of $y^{(i)}$ used when $d=2$.

\section{Can Theorem 1.1 Be strengthened?}

It seems very likely that the condition that $P_{\mathbb{Z}}(Z)$ has no synchronizing word could be weakened, perhaps even to the natural condition that $P_{\mathbb{Z}}(Z)$ be nonsofic.

Question 4.1. Is it true that if $Z$ is a $\mathbb{Z}^{d}$ shift space which is block gluing in directions $\overrightarrow{e_{2}}, \ldots, \overrightarrow{e_{d}}$ with $P_{\mathbb{Z}}(Z)$ nonsofic, then $Z$ is nonsofic?

A slightly weaker version of Question 4.1 was posed by Emmanuel Jeandel for $d=2([9])$.

Question 4.2. Is it true for any $\mathbb{Z}$ shift space $X$ that $X^{\mathbb{Z}}:=\left\{y \in A^{\mathbb{Z}^{2}}\right.$ : all rows of $y$ are in $X$ \} is sofic if and only if $X$ is sofic?

To address this possibility, we will examine the proof of Theorem 1.1 a bit more deeply. (We focus on the $d=2$ case treated in detail in the proof, though our analysis can apply to any $d>1$.) The contradiction derived in the proof of Theorem 1.1 (under the assumption that $Z$ was sofic and block gluing in the $\overrightarrow{e_{2}}$ direction while $P_{\mathbb{Z}}(Z)$ had no synchronizing word) was essentially a set-theoretic obstruction. Specifically, in the last paragraph of the proof, we essentially showed the following fact:

Proposition 4.3. For any $m$, there exists $n$ such that for any $C$, there exists $p$ so that there does not exist a collection of $\mathrm{Cm}^{p}$ sets $\left\{B_{i}\right\}_{i \in\left[1, \mathrm{Cm}^{p}\right]}$ for which every one of the $n^{p}$ different Cartesian products $\prod_{j=1}^{p}\left[1, i_{j}\right], i_{j} \in[1, n]$, can be written as a union of the form $\bigcup_{i \in I} B_{i}$. 
In our proof, we needed Proposition 4.3 for $m=k^{2 L}$ and $C=k^{2 N}$ (which depended on $m$ ), and used $n=k^{2 L}+1$. The sets $B_{i}$ were the sets $U(\delta)$, and the sets $\left[1, i_{j}\right], i_{j} \in[1, n]$, represented intersections of distinct follower sets from $P_{\mathbb{Z}}(Z)$ with a distinguished collection $\left\{y^{(j)}\right\}$ of words, and were obtained from the assumption that $P_{\mathbb{Z}}(Z)$ had no synchronizing word. We point out that $\mathbb{Z}$ shift spaces with finitely many follower sets are known to be sofic. (See [11] for a proof.) This means that even under the much weaker hypothesis that $P_{\mathbb{Z}}(Z)$ were nonsofic, we would still get the existence of infinitely many distinct follower sets and could still attempt to obtain a similar set-theoretic obstruction.

However, we would no longer be able to achieve any particular structure on the sets inside the Cartesian products. The set-theoretic obstruction we would need would look like the following:

Possible Proposition 1. For any $m$, there exists $n$ such that for any $C$, there exists a $p$ so that for $A N Y$ distinct finite sets $A_{1}, \ldots, A_{n}$, there does not exist a collection of $\mathrm{Cm}^{p}$ sets $\left\{B_{i}\right\}_{i \in\left[1, C m^{p}\right]}$ so that every one of the $n^{p}$ different Cartesian products $\prod_{j=1}^{p} A_{i_{j}}, i_{j} \in[1, n]$, can be written as a union of the form $\bigcup_{i \in I} B_{i}$.

If Possible Proposition 1 were true, then we could strengthen Theorem 1.1 to the case where $P_{\mathbb{Z}}(Z)$ is only assumed to be nonsofic. Unfortunately, it is not.

Proposition 4.4. Possible Proposition 1 is false. Specifically, for every $n$ and $p$, there exist finite sets $A_{1}, \ldots, A_{n}$ and a collection of $\lceil\log (n+1)\rceil 8^{p}$ sets $\left\{B_{i}\right\}_{i \in\left[1,[\log (n+1)] 8^{p}\right]}$ so that every one of the $n^{p}$ different Cartesian products $\prod_{j=1}^{p} A_{i_{j}}, i_{j} \in[1, n]$ can be written as a union of the form $\bigcup_{i \in I} B_{i}$.

Proof. Choose any $n$ and $p$. For now we assume that $n$ is even. Our sets $A_{i}$, $1 \leq i \leq n$, will be $[1, n] \backslash\{i\}$. We will define a collection of sets $\left\{B_{i}\right\}_{i \in\left[1,\lceil\log (n+1)\rceil 8^{p}\right]}$, where each $B_{i}$ will be a Cartesian product of sets $C_{j}^{(i)}, 1 \leq j \leq p$, each of cardinality $0.5 n$, to be chosen soon. For any $\vec{i}=\left(i_{1}, \ldots, i_{p}\right) \in[1, n]^{p}$, to show that $\prod_{j=1}^{p} A_{i_{j}}$ is a union of the form $\bigcup_{i \in I} B_{i}$, it suffices to show that for any $\vec{k}=\left(k_{1}, \ldots, k_{p}\right) \in[1, n]^{p}$ with $k_{m} \neq i_{m}$ for all $m$, there exists $B_{i}$ which contains $\vec{k}$, but is contained in $\prod_{j=1}^{p} A_{i_{j}}=\prod_{j=1}^{p}\left([1, n] \backslash\left\{i_{j}\right\}\right)$. Since each $B_{i}$ will be chosen to be a Cartesian product, this is equivalent to saying that there is a $B_{i}$ containing $\vec{k}$ but not $\vec{i}$. Therefore, to prove the theorem, it suffices to show that for any $\vec{k}, \vec{i} \in[1, n]^{p}$ with $k_{m} \neq i_{m}$ for all $m$, there exists $B_{i}=\prod_{i=1}^{p} C_{j}^{(i)}$ containing $\vec{k}$ but not $\vec{i}$.

For each $i \in\left[1,\lceil\log n\rceil 8^{p}\right]$ and $j \in[1, p]$, independently choose $C_{j}^{(i)} \subset[1, n]$ of cardinality $0.5 n$ uniformly at random, and for each $i \in\left[1,\lceil\log n\rceil 8^{p}\right]$, define $B_{i}=\prod_{j=1}^{p} C_{j}^{(i)}$. Then for any $\vec{k}, \vec{i} \in[1, n]^{p}$ with $k_{m} \neq i_{m}$ for all $m$, the probability that a specific $B_{i}$ contains $\vec{k}$ but not $\vec{i}$ is exactly $4^{-p}$. Since the $B_{i}$ are independently chosen, the probability that none of the $B_{i}$ has this property is $\left(1-4^{-p}\right)^{\lceil\log n\rceil 8^{p}}=$ $\left(1-4^{-p}\right)^{\left(4^{p}\right)\lceil\log n\rceil 2^{p}}<e^{-2^{p} \log n}=n^{-2^{p}}$. But then, by summing over all choices for $\vec{k}$ and $\vec{i}$, the probability that there exist any choices of $\vec{k}$ and $\vec{i}$ so that none of the $B_{i}$ contains $\vec{k}$ but not $\vec{i}$ is less than 1 , meaning that with nonzero probability, every product $\prod_{j=1}^{p} A_{i_{j}}$ can be written as a union of the form $\bigcup_{i \in I} B_{i}$. Since this happens with nonzero probability, there must be some choice of the $B_{i}$ for which it does happen, completing the proof for even $n$. 
If $n$ is odd, then $n+1$ is even, and so there are $\lceil\log (n+1)\rceil 8^{p}$ sets $B_{i} \subseteq[1, n+1]^{p}$ such that all products $\prod_{j=1}^{p} A_{i_{j}}, 1 \leq i_{j} \leq n+1$, can be written as unions of the form $\bigcup_{i \in I} B_{i}$. Restricting each $B_{i}$ to $[1, n]^{p}$ gives a collection of size at most $\lceil\log (n+1)\rceil 8^{p}$ satisfying the conditions of the theorem.

This provides a contradiction to Possible Proposition 1 for $m=8$ and $C=$ $\lceil\log (n+1)\rceil$.

Of course this does not by itself preclude extending Theorem 1.1 to the general nonsofic case, since the specific case $A_{i}=[1, n] \backslash\{i\}$ might never occur as intersections of follower sets with a collection $\left\{y^{(j)}\right\}$ of words. Unfortunately, there is at least one example of a nonsofic subshift whose follower set structure is of this form.

Example 4.5. Define the reverse context-free shift $X$ on the alphabet $\{a, b, c\}$ by the forbidden configurations $\mathcal{F}=\left\{c a^{n} b^{n} c\right\}_{n \in \mathbb{N}}$. Then the following are descriptions of all follower sets of $X$ (we describe the complements of the follower sets, as it is more concise):

$$
\begin{aligned}
(F(\varnothing))^{c} & =\varnothing \\
\left(F\left(c a^{i}\right)\right)^{c} & =\left\{a^{j} b^{i+j} c \ldots: j \geq 0\right\} \text { for any } i \geq 0, \\
\left(F\left(c a^{i} b^{j}\right)\right)^{c} & =\left\{b^{i-j} c \ldots\right\} \text { for any } i \geq 0 \text { and } j \leq i .
\end{aligned}
$$

$X$ has infinitely many follower sets, so it is nonsofic. However, the word $c$ is synchronizing for $X$, and so Theorem 1.1 does not let us show that $X^{\mathbb{Z}}$ is nonsofic.

We note that for any two unequal follower sets $F(u) \neq F\left(u^{\prime}\right)$ in $X,(F(u))^{c} \cap$ $\left(F\left(u^{\prime}\right)\right)^{c}=\varnothing$. This means that we are essentially in the "bad situation" from Proposition 4.4 for any collections $\left\{F\left(x^{(k)}\right)\right\}$ of distinct follower sets and $\left\{y^{(j)}\right\}$ of words in $L(X)$, each intersection $A_{k}:=F\left(x^{(k)}\right) \cap\left\{y^{(j)}\right\}$ is of the form $\left\{y^{(j)}: j \in\right.$ $\left.[1, n] \backslash J_{k}\right\}$, where the sets $J_{k}$ are pairwise disjoint. This allows for the construction of a collection of at most $(2 p \log (n+1)) 4^{p}$ sets for which each product $\prod_{j=1}^{p} A_{i_{j}}$ is a union of the form $\bigcup_{i \in I} B_{i}$; simply take the collection $\left\{B_{i}\right\}$ from Proposition 4.4 and identify each $k \in[1, n]$ with the entire set $\left\{y^{(j)}: j \in J_{k}\right\}$. (If the union of all $J_{k}$ is a proper subset of $[1, n]$, then simply add to each $B_{i}$ the set of $p$-tuples $\left(y^{\left(j_{1}\right)}, \ldots, y^{\left(j_{p}\right)}\right)$ in which one of the $y^{\left(j_{m}\right)}$ is not in any $J_{k}$.)

At the moment, we do not see how to extend the ideas in the proof of Theorem 1.1 to address the case where $P_{\mathbb{Z}}(Z)$ is only assumed to be nonsofic, or even the specific case where $P_{\mathbb{Z}}(Z)$ is the reverse context-free shift. One idea would be to show some additional properties about the sets $U(\delta)$ so that a weaker version of Possible Proposition 1, where the sets $B_{i}$ are assumed to have these additional properties, is true. (For instance, the sets $B_{i}$ constructed in the proof of Proposition 4.4 were all products; perhaps the $U(\delta)$ are far from being products in some sense, and perhaps a version of Possible Proposition 1 where the sets $B_{i}$ are assumed to be far from being products in this sense is true.) However, the same phenomena behind the usual undecidability issues for $\mathbb{Z}^{d}$ SFTs might make it very difficult to get such explicit information about the sets $U(\delta)$.

\section{ACKNOWLEDGEMENTS}

The author would like to thank the anonymous referee for useful comments and suggestions and would also like to acknowledge the support of the University of British Columbia, where the author was a postdoc during much of this work. 


\section{REFERENCES}

[1] N. Aubrun and M. Sablik, Simulation of effective shift spaces by two-dimensional subshifts of finite type, Acta Appl. Math., to appear.

[2] R. Berger, The undecidability of the domino problem, Mem. Amer. Math. Soc. 66 (1966). MR0216954(36:49)

[3] M. Boyle, R. Pavlov, and M. Schraudner, Multidimensional sofic shifts without separation and their factors, Trans. Amer. Math. Soc. 362 (2010), 4617-4653. MR2645044 (2011g:37037)

[4] A. Desai, Subsystem entropy for $\mathbb{Z}^{d}$ sofic shifts, Indagationes Mathematicae 17 (2006), no. 3, 353-360. MR2321105 (2009h:37020)

[5] B. Durand, A. Romashchenko, and A. Shen, Fixed point sets and their applications, J. Comput. System Sci., to appear.

[6] N. Pytheas Fogg, Substitutions in Dynamics, Arithmetics and Combinatorics, Lecture Notes in Mathematics 1794, Springer, Berlin, 2002. MR1970385(2004c:37005)

[7] M. Hochman, On the dynamics and recursive properties of multidimensional symbolic systems, Inventiones Mathematicae 176 (2009), no. 1, 131-167. MR2485881(2009m:37023)

[8] F. Hahn and Y. Katznelson, On the entropy of uniquely ergodic transformations, Trans. Amer. Math. Soc. 126 (1967), 335-360. MR0207959 (34:7772)

[9] E. Jeandel, personal communication.

[10] A. Johnson, S. Kass, and K. Madden, Projectional entropy in higher dimensional shifts of finite type, Complex Systems 17 (2007), no. 3, 243-257. MR2373706 (2008m:37028)

[11] D. Lind and B. Marcus, Introduction to symbolic dynamics and coding, Cambridge University Press, Cambridge, 1995. MR 1369092 (97a:58050)

[12] R. Pavlov and M. Schraudner, Projectional subdynamics of $\mathbb{Z}^{d}$ shifts of finite type, submitted.

[13] P. Walters, An Introduction to Ergodic Theory, Graduate Texts in Mathematics 79, SpringerVerlag, Berlin, 1982. MR648108 (84e:28017)

[14] H. Wang, Proving theorems by pattern recognition. II, AT\&T Bell Labs. Tech. J. 40 (1961), 1-41.

Department of Mathematics, University of Denver, 2360 S. Gaylord Street, Denver, Colorado 80208

E-mail address: rpavlov@du.edu

$U R L$ : www.math.du.edu/ rpavlov/ 